\title{
A prospective investigation into the nutritional status of Irish oncology outpatients: prevalence of malnutrition, cachexia, sarcopenia and impact on quality of life
}

\author{
S. J. Cushen ${ }^{1}$, D. G. Power ${ }^{2}$, P. McEneaney ${ }^{3}$, L. Daly ${ }^{1}$ and A. M. Ryan ${ }^{1}$ \\ ${ }^{1}$ School of Food and Nutritional Sciences, University College Cork, Republic of Ireland, ${ }^{2}$ Dept Medical Oncology, \\ the Mercy University Hospital, Republic of Ireland and ${ }^{3}$ Dept of Radiology, the Mercy University Hospital, Cork, \\ Republic of Ireland
}

Malnutrition is a significant factor in predicting cancer patients' quality of life (QoL), tolerance to treatment, and overall survival ${ }^{(1)}$. Our study describes prevalence of malnutrition, cachexia and sarcopenia and their impact on QoL for the first time in Irish cancer patients undergoing chemotherapy in a regional cancer centre.

A prospective cross-sectional study of adult cancer patients undergoing chemotherapy was conducted. Malnutrition Universal Screening Tool scores (MUST) and QoL (EORTC QLQ-C30) were measured. Cancer cachexia was defined as weight loss (WL) $>5 \%$ over the past 6 months or WL $>2 \%$ in combination with a Body Mass Index $(\mathrm{BMI})<20 \mathrm{~kg} / \mathrm{m}^{2}$ or sarcopenia $^{(2)}$. Skeletal muscle was measured by CT scan in all patients. Sarcopenia was defined using published cut offs ${ }^{(3)}$.

432 patients receiving chemotherapy (276 male), with a mean age of 63.5(SD 13.8) were included. The percentage of patients with colorectal cancer was highest $(30 \cdot 8 \%)$ followed by upper gastrointestinal cancer $(19 \cdot 7 \%)$, and hepatobillary cancer $(10 \cdot 6 \%)$. $53 \cdot 6 \%$ were overweight or obese (BMI $\left.>25 \mathrm{~kg} / \mathrm{m}^{2}\right)$. Sarcopenia was present in $42.4 \%$ (32.3\% of overweight and obese groups). Based on MUST scores $36.4 \%$ were at medium to high risk of malnutrition. WL $(\geqslant 5 \%)$ in the previous 6 months was highest in GI tumours $(24.9 \% ; \mathrm{P}<0.001)$ and was significantly associated with loss of appetite $(\mathrm{P}<0.001)$, adverse global QoL $(\mathrm{P}=0.025)$ diarrhoea $(\mathrm{P}=0.018)$ and nausea and vomiting $(\mathrm{P}=0.016)$. Similarly cachexia was highest in hepatobillary $(65 \cdot 2 \%)$ and upper GI tumours $(64 \cdot 3 \%)$ and was associated with poorer performance status. Cachexia was associated with adverse global QoL $(\mathrm{P}=0.003)$, poor appetite $(\mathrm{P}<0.001)$ and nausea and vomiting $(\mathrm{P}<0.001)$.

The prevalence of malnutrition, cachexia and sarcopenia is very high in Irish cancer patients but is disguised by a mantel of adipose tissue. Site of primary tumour appears to be associated with significant weight loss, nutritional risk and QOL. Early screening by MUST and CT assessment of body composition would allow for prompt nutrition intervention in these patients.

1. Jacquelin-Ravel N \& Pichard C (2012) Crit Rev Oncol Hematol 84, 37-46.

2. Fearon K, Strasser F, Anker SD et al. (2011) Lancet Oncol 12, 489-495.

3. Mourtzakis M, Prado CM, Lieffers JR et al. (2008) Appl Physiol Nutr Metab 33, 997-1006. 